\title{
Digestive surgeseriy
}

Abdominal discomfort/pain 453

Acute liver failure 254

- pancreatitis 234

Adenocarcinoma 130

Adenoma 182

Adjuvant chemoradiotherapy 321

Anal fistulas 91

Antiangiogenesis, gastrointestinal tumors 282

Antibiotic prophylaxis, surgical patients 234

Antifibrinolytics, liver transplantation 265

Appendix 163

Bacterial translocation 234

Biliary colic 95

Bioartificial liver 254

Blood loss 265

- transfusion 265

Bowel necrosis 375

Cancer 80, 163, 182, 353

- gene therapy, strategies 222

Carcinoma, rectal 41

Caudate lobe 143

Cervical esophageal adenocarcinoma 107

Chemoradiotherapy 353

Chemotherapy 353, 401

Cholangiocarcinoma 464

Cholecystectomy 426

Chronic pancreatitis 55, 340

c-KIT 364

Classification, haemorrhoids 26

Colitis, acute 135

Colon surgery 168

Colonic squamous cell carcinoma 371

Colorectal cancer 16, 174, 276, 401

- -, emergency surgery 174

- -, prognosis 276

- $\quad$, intestinal obstruction 174

- $\quad$, treatment 174

- malignancy 34

Complication registration 168

Complications 182

Computed tomography 80

Crohn's disease 135

Cytokeratin 364

Cytomegalovirus 464
Digestive system 346

Distal oesophagus 130

Diverticulitis 100, 419

Dorsal approach 86

Duodenal diverticuli 198

Duodenum-preserving pancreatic head resection 340

Ectopic pancreas 377

Elderly patients 100

Endocrine tumours 157

Endoscopic microsurgery, transanal 6

- surgery 313

Endoscopy 295

Enhanced recovery 346

Esophageal cancer, optimizing gene therapy 222

Evidence-based medicine 346

Excision and open wound 459

Familial adenomatous polyposis 69

Fibrin glue 91

5-Fluorouracil 191, 401

Gallbladder neoplasms 440

Gastrointestinal cancer 282

Gastro-oesophageal junction 130

Gene delivery methods 222

- -, esophageal cancer 222

Genomics 276

Haemorrhoids, aetiology 26

Hartmann procedure 419

Hemostasis, liver transplantation 265

Hepatic progenitor cell 364

Hepatitis C virus 432

Hepatocellular carcinoma 364

Hepatocytes 254

Heterotopic gastric mucosa 107

Hypercalcemia 371

Hyperleukocytosis 371

IIeal pouch anal anastomosis 69

Immune system, infection prevention 234

Incontinence 1,91

Infected pancreatic necrosis 446

Inflammatory bowel disease 135

Inlet patch 107

Intrahepatic cholangiocarcinoma 432
Irinotecan 401

Irradical resections 321

Laparoscopic cholecystectomy 95

- surgery 50, 135

- -, indications/contra-indications 34

- techniques 34

- ultrasound 55

Laparoscopy 34, 55, 313, 426, 440

Left colonic peritonitis 100

Liver metastases 16

- resection 143, 432

- segmental anatomy 143

- transplantation 254, 265

- tumor(s) 143, 245

Local excision 86

- -, rectal cancer 6

Malignant insulinoma 377

- _ jaundice 295

Mesorectal excision 41

Minimal residual disease, colorectal cancer 276

Morbidity and mortality, outcome and risk factors 174

Mouse model 16

Neoadjuvant treatment 191

Nonfunctioning neuroendocrine neoplasms, pancreas 453

Obstructing colorectal carcinoma 174

Octreotide scintigraphy 377

Oesophageal adenocarcinoma 130

- cancer 63

- surgery 130

Optical imaging 16

Optimal perioperative care 346

Oxaliplatin 401

Palliative drainage 295

Pancreas 80, 157, 353

- neoplasms 453

Pancreatic 353

- abscess 446

- - cancer 191, 321

- - fistula 446

- pseudocyst 340

Papillitis 464 
Parastomal hernia 152

Perforated 100

Perforation 198

Physical function 63

Pilonidal sinus 459

Polypropylene mesh prosthesis 152

- ring 152

POSSUM 419

Posterior approach 86

Post-operative complications 353

Postoperative enteral feeding 375

p-POSSUM 419

Primary appendiceal carcinoma 163

Probiotics 234

Proteomics 276

Proximate Linear Cutter 100, stoma prolapse repair 306

Pseudocyst-jejunostomy 340

Pyloric exclusion 198

Quality control 168

- of life 63, 295
Radiofrequency 459

- , liver tumors 245

Radiotherapy 41

Randomized controlled trial 353

Range of motion 63

Rectal cancer 6

Rectum 86, 182

Rectus abdominis muscle 152

Recurrence 459

Resection 80, 353

Retromesenteric approach 50

Right colon 50

Robot 313

Robot-assisted surgery 313

Robotic surgery 426

Sacral nerve stimulation 1

Small animal imaging 16

Spirometry 63

Stoma prolapse repair 306

Stoma-related complications 306

Surgery 80, 86, 353
Surgical management, haemorrhoids 26 - procedure 346

Survival, surgery 157

Thoracoabdominal resection 63

Tissucol 91

Transanal endoscopic microsurgery 182

- excision 6

Tube jejunostomy 375

Tumor model 16

Tumour markers 80

Ulcerative colitis 69

Vascular endothelial growth factor, gastrointestinal oncology 282 